\title{
Hormone Use is Associated with Lymphovascular Invasion in Breast Cancer
}

\author{
Margaretha Loof-Johanson ${ }^{1 *}$, Lars Brudin ${ }^{1,2}$, Marie Sundquist ${ }^{3}$, Carl Edvard \\ Rudebeck $^{4}$
}

\begin{abstract}
Background: Risk of developing breast cancer increases with short breastfeeding and the use of hormones. The prognosis of breast cancer is better if the tumours are hormone receptor positive. Since breast feeding affects estrogen and progesterone receptors, we wanted to investigate how such reproductive factors as breastfeeding and the use of hormones interact with known prognostic markers and specific tumour characteristics in women with breast cancer. Materials and Methods: A total of 250 women treated for breast cancer from a larger cohort completed a questionnaire on breastfeeding, number and age at births and use of hormones. A logistic regression analysis was made to search for connections between known prognostic markers on the one hand (type of cancer, grade, tumor size, estrogen receptor and progesterone receptor, lymphovascular invasion and DNA-ploidy) and reproductive data, breastfeeding, and hormone use on the other. Results and Conclusions: Hormone use, but not breastfeeding, was significantly associated, also on multivariate analysis, with the prognostic variable lymphovascular invasion, connected to a worse prognosis. No other hormone use or breast feeding correlations with prognostic variables were found.
\end{abstract}

Keywords: Breastfeeding - childbirth - breast cancer - prognostic factors in breast cancer - LVI - NHG - DNA - ER/PR

Asian Pac J Cancer Prev, 17 (3), 1507-1512

\section{Introduction}

Risk of developing breast cancer increases with short breastfeeding and use of hormones (Collaborative Group on Hormonal Factors in Breast Cancer 2002; Anothaisintawee et al., 2013; Antoine et al., 2014; Scneider et al., 2014). Anothaisintawee et al., 2013 assumed that the etiology of breast cancer can be explained by two mechanisms of differentiation and proliferation of breast epithelial cells mediated by hormonal factors. They found associated adverse risk factors for both mechanisms in the use of oral contraceptives (OC) and hormonal replacement therapy (HRT). The association with breastfeeding as a significant and positive influence factor against breast cancer decreased the risk with $28 \%$ if breastfeeding $\geq 12$ months.

Li et al. found, 2013 that breastfeeding generates a lower risk of triple-negative breast cancer, $(\mathrm{TNBC}=$ negative ER/PR and Her2 breast cancer) supported by both their case-control studies $(\mathrm{p}=0.02)$ and case-case comparisons $(p=0.01)$.Their observations are consistent with the results of the majority of studies that have assessed these relationships and breastfeeding is the most consistently identified factor to be differentially associated with risk of triple- negative cancer compared to other major molecular subtypes of the disease. The biological mechanisms for this is largely unknown.

Phipps et al., 2011, present different results. They found that nulliparity was associated with decreased risk of triple-negative breast cancer but increased risk of ER+ breast cancer. Among parous women, the number of births was positively associated with risk of triple-negative disease for three births or more vs one birth- and inversely associated with risk of ER+ disease. Ages at menarche and menopause were modestly associated with risk of ER+ but not triple-negative breast cancer; breastfeeding and oral contraceptive use were not associated with either subtype.

Work et al. assume, 2014, that the ER / PR negative breast cancer has a poorer prognosis compared with other subtypes of breast cancer and that breast-feeding $>12$ months can reduce the risk of all subtypes and with the strongest effect on ER- / PR-.

Our knowledge of how reproductive factors and the use of hormones affect the risk of developing breast cancer is greater than our knowledge of how the prognosis of the breast cancer is affected. Here we have a large need for research (Kwan et al., 2015).

Our aim was to evaluate if reproductive factors or use of hormones were associated with any specific tumour characteristics. Since breast feeding and the use of

${ }^{1}$ Department Medical and Health Sciences, University of Linkoping, ${ }^{2}$ Department of Clinical Physiology, ${ }^{3}$ Department of Surgery, County Hospital, Kalmar, Sweden, ${ }^{4}$ Department of Community Medicine, University of Tromso, Tromso, Norway *For correspondence: 0485.40311@telia.com 
hormones affect estrogen and progesterone receptors and indirectly the risk for illness, we also wanted to examine how breastfeeding and the use of hormones interact with known prognostic markers in women with breast cancer.

\section{Materials and Methods}

This study investigates possible associations between prognostic markers and specific tumour characteristics on the one hand, and reproductive data as breast-feeding, and hormone use on the other. We have used a questionnaire in a subgroup of 250 women treated for breast cancer from the Southeast Swedish Breast Cancer Registry (Sundquist, 2000).

\section{Study population}

From 1988 to 1992, 630 women, 25-74 years of age, were treated for primary breast carcinoma without distance metastases in the Counties of Kalmar and Ostergotland

(Sundquist, 2000). At the time of the interview 275/630 women (44\%), had died and another 10 women in terminal care of the disease were excluded (1.6\%). Questionnaires were sent to 345 women and 250/345 (72\%) were returned.

The Research Ethics Committee of Linkoping University Hospital approved the study and informed consent was obtained from each participant.

\section{Prognostic markers (dependent variables)}

Histological type: The tumours were classified according to the World Health Organisation, WHO. First, all histological types represented in the cohort were considered in the analyses but as ductal (69\%) and lobular (20\%) tumours dominated, the parameter was finally dichotomized into ductal and lobular.

Tumour size: Tumour size was retrieved from the original pathology reports.

Lymph node stage (LNS): Lymph node stages (LNS) were classified as: $1=$ no positive lymph nodes, $2=1-3$ positive lymph nodes or $3=$ more than 3 positive lymph nodes.

Nottingham histological grade (NHG): Grading was performed according to the protocol of CW Elston. (Elston et al., 1991; Ellis et al., 1992) in grade 1-3.

Lymphovascular invasion (LVI): LVI was assessed simultaneously with grading and defined as the presence of malignant cells within a vessel in the periphery of the tumour. $(=1)$, or otherwise negative $(=0)$ (Pinder et al., 1994).

DNA- ploidy (PLOIDY): The DNA ploidy was categorized as 1 if diploid, otherwise as 0 (nondiploid) (Kallioniemi et al., 1987; Stal et al., 1992; Pinder et al., 1994; Dieterich et al., 1995).

Estrogen- and progesteronreceptors: Estrogen positive tumours were is stated as positive or negative. Cut off for ER and PR positivity is $0.3 \mathrm{fmol} /$ microgram (Dieterich et al., 1995).

\section{Parameters from the questionnaire (independent variables)}

Reproductive data: Age at first child (AFC) was categorized as 0 if AFC $<23$ years (median value for all women having at least one child), otherwise as 1 including patients with no children. Time between last childbirth to cancer diagnosis is categorized as $\leq 26$ yrs and $>26$ yrs, respectively (median) in the logistic regression analysis. Number of children was categorized as follows: 1=no children (only four of these women having had either an abortion $(n=2)$ or miscarriage $(n=2), 2=$ one child, $3=2$ or more children. Breastfeeding time (BFT) was dichotomized as no breastfeeding or breastfeeding. Abortions and miscarriages $0=$ none otherwise $1=$ yes .

Hormone use: Hormone use included contraceptive pills (e.g. low-dose pills), combination products adhesive patches, contraceptive injections, hormone coil, and hormone replacement therapy. It was not possible from the questionnaire to differentiate between contraceptives and hormone replacement therapy. Nevertheless, a subgroup analysis was made with women $<48$ years of age based on the assumption that they were using only contraceptives. Gestagen, oestrogen and a combination of these were primarily analysed separately (see results) but since no differences were found, all three groups were lumped together and categorized as hormones and no hormones respectively. Duration of treatment was also assessed (Ppyear).

\section{Data analysis and statistics}

The material is selected, only women alive at the time of the interview were able to participate. Relevant conclusions could, therefore, only be made if hormone use or reproductive factors did not interact with the prognostic markers to influence prognosis. For example, if the prognosis of adverse prognostic markers were partly due to hormone use, a selection of women with bad prognostic markers and non-hormone use might occur among the survivors. This would then blur an existing correlation between hormone use and bad prognostic markers in the entire cohort. Similarly, with an increasing time-lapse between date of diagnosis and date of interview, the combination of bad prognostic markers and hormone use would become more biased. Therefore, the material was initially split into those who had had their breast cancer diagnosis early in relation to the date of the interview (<median diagnosis date, 24-aug-89) and late, respectively, and the two groups were compared according to hormone use (no/yes) using logistic regression, adjusting for age ( $\leq$ or $>50$ yrs) and time of diagnosis (before or after Aug 1989). Since no convincing evidence of interaction could be demonstrated, further multivariate logistic regression analyses were used to assess correlations between the dichotomous dependent parameters (cancer type and prognostic markers) on the one hand, and the independent parameters (hormone use (no/yes) and the various reproductive data) on the other.

Regarding abortions and miscarriages, analyses were made both including and excluding these women but the results were very similar. Hence patients with abortions and miscarriages were not accounted for, apart from the categorization of total breastfeeding time (see above).

The material was divided into four categories for descriptive purposes; breastfeeding women (no/yes) using hormones (no/yes) and the differences between these 
groups were descriptively analysed using Fishers exact test for frequencies

\section{Results}

\section{Main Results}

Hormone use, but not breastfeeding, was significantly associated to lymphovascular invasion (LVI), and to a worse prognosis. Table 3 .

\section{Descriptive statistics}

Table 1 shows questionnaire data categorized according to hormone use and breastfeeding. Some 146 women (58\%) had not used hormones at all and 40 of the 250 women (16\%) in the study had not breastfed. The time of breastfeeding did not differ between non-hormone and hormone users.

The women in our material diagnosed late in life were already too old to be offered oral contraception when these were introduced in Sweden in 1964 and so most of them were, when HRT was introduced on a broad front. This explains why hormone users were younger at the time of diagnosis ( $\mathrm{p} \leq 0.001$, Mann-Whitneys U-test) and, as expected, this difference disappeared for those under 50 (Table 1). Non-breastfeeding women using hormones showed a significantly shorter time between their last child's birth and the cancer diagnosis compared to nonhormone users irrespective of breastfeeding $(\mathrm{p}=0.001)$ (Table 1).

The number of subjects using oestrogen was $71(28 \%)$, gestagen $8(3 \%)$, a combination of these $14(6 \%)$, and no hormones $157(63 \%)$. There were no differences between the three hormone categories concerning prognostic markers (Fishers exact test). The gestagen, oestrogen and combination groups were, therefore, combined in the analyses. Breastfeeding women had, compared with non-breastfeeding women, more frequent use of oral contraceptives $(\mathrm{p}<0.001$; Table 1$)$. This could possibly be due to the fact that sexually inactive women abstain from usage of oral contraceptives.

Table 1. Questionnaire Data Categorized According to Breastfeeding and Hormone Use

\begin{tabular}{|c|c|c|c|c|c|}
\hline \multirow{2}{*}{ Parameters } & \multicolumn{2}{|c|}{ No breast feeding } & \multicolumn{2}{|c|}{ Breast feeding } & \multirow{2}{*}{ p-value* } \\
\hline & No hormones & Hormones & No hormones & Hormones & \\
\hline $\mathrm{N}(\mathrm{N} \text { for women } \leq 50 \mathrm{yrs})^{* *}$ & $28(5)$ & $12(7)$ & $118(21)$ & $92(47)$ & - \\
\hline $\begin{array}{l}\text { Age at carcinoma diagnosis (median; } \\
\text { yrs) }\end{array}$ & $61.1(43-74)$ & $49.0(33-61)$ & $63.1(35-75)$ & $49.6(31-69)$ & $<0.001$ \\
\hline Do: for women $\leq 50 \mathrm{yrs}$ & $48.7(43-50)$ & $46.8(33-49)$ & $44.4(35-49)$ & $43.7(31-50)$ & 0.181 \\
\hline Age at first child (median; yrs) & $23.0(21-32)$ & $30.0(20-36)$ & $24.0(17-40)$ & $22.8(16-39)$ & 0.210 \\
\hline Age at first child $<23$ yrs & $3(38 \%)$ & $1(17 \%)$ & $51(43 \%)$ & $48(52 \%)$ & 0.344 \\
\hline Time between last child and ca op (yrs) & $36(16-62)$ & $16(2-28)$ & $33(1-62)$ & $21(-4-51)$ & $<0.001$ \\
\hline Do: for women $\leq 50 \mathrm{yrs}$ & $17.4(16-19)$ & $14.8(2-17)$ & $14.8(1-38)$ & $15.6(-4-38)$ & 0.782 \\
\hline $\begin{array}{l}\text { Breastfeeding time per child (median; } \\
\text { months) }\end{array}$ & - & - & $5.0(0-14)$ & $5.0(0-14)$ & 0.651 \\
\hline $\begin{array}{l}\text { Totalbreast feeding time (median; } \\
\text { months) }\end{array}$ & - & - & $10.0(1-46)$ & $10.0(1-96)$ & 0.505 \\
\hline Miscarriages & $2(7 \%)$ & $3(25 \%)$ & $26(22 \%)$ & $22(24 \%)$ & 0.433 \\
\hline Abortions & $2(7 \%)$ & $2(17 \%)$ & $3(3 \%)$ & $14(15 \%)$ & 0.001 \\
\hline Ppyear & - & $3.5(0-15)$ & - & $5.0(0-25)$ & $<0.001$ \\
\hline
\end{tabular}

*Only hormones vs. no hormones analyzed (Fishers' exact test for categorized and Mann-Whitney for continuous variables), $* *$ Concerning variables involving children $\mathrm{N}$ is correspondingly lower

Table 2. Tumour Characteristics Stratified According to Breastfeeding and Hormone Use

\begin{tabular}{|c|c|c|c|c|c|}
\hline \multirow{2}{*}{ Parameters } & \multicolumn{2}{|c|}{ No breast feeding } & \multicolumn{2}{|c|}{ Breast feeding } & \multirow{2}{*}{ p-value* } \\
\hline & No hormones & Hormones & No hormones & Hormones & \\
\hline $\mathrm{N}(\mathrm{N}$ for women $\leq 50 \mathrm{yrs})$ & $28(5)$ & $12(7)$ & $118(21)$ & $92(47)$ & - \\
\hline Tumour type & & & & & $0.260 * *$ \\
\hline Lobular & $4(14 \%)$ & $3(25 \%)$ & $30(25 \%)$ & $14(15 \%)$ & \\
\hline Ductal & $22(79 \%)$ & $8(67 \%)$ & $77(65 \%)$ & $65(71 \%)$ & 0.012 \\
\hline NHG 3 & $11(39 \%)$ & $3(25 \%)$ & $24(20 \%)$ & $38(41 \%)$ & 0.337 \\
\hline Do: for women $\leq 50 \mathrm{yrs}$ & $1(20 \%)$ & $2(29 \%)$ & $8(38 \%)$ & $24(51 \%)$ & 0.701 \\
\hline LVI positive & $1(4 \%)$ & $2(17 \%)$ & $13(11 \%)$ & $20(22 \%)$ & 0.017 \\
\hline Do: for women $\leq 50 \mathrm{yrs}$ & $0(0 \%)$ & $1(14 \%)$ & $3(14 \%)$ & $10(21 \%)$ & 0.531 \\
\hline Oestrogen receptor pos & $12(63 \%)$ & $8(100 \%)$ & $77(83 \%)$ & $47(68 \%)$ & 0.226 \\
\hline Progesterone receptor pos & $10(53 \%)$ & $7(88 \%)$ & $63(68 \%)$ & $48(70 \%)$ & 0.429 \\
\hline Nondiploid & $12(67 \%)$ & $5(63 \%)$ & $41(49 \%)$ & $39(64 \%)$ & 0.158 \\
\hline
\end{tabular}

* Only hormones vs. no hormones analyzed (Fisher's exact test), ** Only ductal/lobular statistically analyzed 
Table 3. Results from Multivariate Logistic Regression with Reproductive Data and Hormone Use as Independent Variables and Established Prognostic Factors for Breast Cancer as Dependent

\begin{tabular}{|c|c|c|c|c|c|}
\hline \multirow{2}{*}{ Parameter } & \multirow{2}{*}{ Total } & \multicolumn{2}{|c|}{ Tumour type* } & \multicolumn{2}{|c|}{ Multivariate logistic regression } \\
\hline & & Lobular & $(\%)$ & OR $(95 \%$ conf int $)$ & $\mathrm{p}$ \\
\hline \multicolumn{6}{|c|}{ Number of children } \\
\hline 0 & 24 & 3 & 13 & 1.00 & \multirow{3}{*}{0.047} \\
\hline 1 & 47 & 10 & 21 & $1.73(1.01-2.96)$ & \\
\hline$\geq 2$ & 152 & 38 & 25 & $2.98(1.01-8.76)$ & \\
\hline \multicolumn{6}{|c|}{ Age at first child } \\
\hline$<23$ & 77 & 12 & 16 & 1.00 & \multirow{2}{*}{0.022} \\
\hline$\geq 23$ & 146 & 39 & 27 & $2.40(1.14-5.06)$ & \\
\hline \multicolumn{6}{|c|}{ Lympho Vascular invasion (LVI) } \\
\hline Parameter & Totalt & Pos & $(\%)$ & & \\
\hline \multicolumn{6}{|c|}{ Hormone treatment } \\
\hline No & 146 & 14 & 10 & 1.00 & \multirow{2}{*}{0.044} \\
\hline Yes & 104 & 22 & 21 & $2.24(1.02-4.93)$ & \\
\hline \multicolumn{6}{|c|}{ Age at first child } \\
\hline$<23$ & 90 & 19 & 21 & 1.00 & \multirow{2}{*}{0.041} \\
\hline$\geq 23$ & 160 & 17 & 11 & $0.47(0.97-0.22)$ & \\
\hline \multicolumn{6}{|c|}{ Estrogen receptor } \\
\hline Parameter & Totalt & Neg & $(\%)$ & & \\
\hline \multicolumn{6}{|c|}{ Time from last child to cancer } \\
\hline$\leq 26$ & 89 & 26 & 29 & 1.00 & \multirow{2}{*}{0.011} \\
\hline$>26$ & 98 & 17 & 17 & $0.34(0.78-0.15)$ & \\
\hline \multicolumn{6}{|c|}{ Nondiploid } \\
\hline Parameter & Totalt & Yes & $(\%)$ & & \\
\hline \multicolumn{6}{|c|}{ Age at first child } \\
\hline$<23$ & 65 & 41 & 63 & 1.00 & \multirow{2}{*}{0.038} \\
\hline$\geq 23$ & 103 & 48 & 47 & $0.50(0.26-0.96)$ & \\
\hline
\end{tabular}

Dependent variables (each dichotomous) adjusted for age at diagnosis and time of diagnosis (before Aug 1989 or after). Only statistically significant associations are included (see text). Except for tumour type, parameters with poorer prognosis are tabled and percentage given (e.g. negative oestrogen receptor or non-diploid tumours) *only ductal and lobular included

Hormone use associated with prognostic variables.

This paper centres on relationships between hormone use in combination with breastfeeding on the one hand, and possible associations with various prognostic parameters on the other. Detailed frequencies are shown in Table 2. All statistically significant interrelationships using multivariate logistic regression (see data analysis) are shown in Table 3. The only multivariate association between hormone use and prognostic variables, was statistically significant to lymphovascular invasion (Table 3 ) and this significance did not change when adjusted for early/late diagnosis ( $p=0.029$; see Table 3 ). Grade 3 was univariately associated to hormone use $(\mathrm{p}=0.012$; see Table 2) but this correlation disappeared when adjusted for age at date of diagnosis.

\section{Other reproductive data and prognostic variable}

Table 3 summarizes the statistically significant relationships, partly published recently (Dieterich et al.1995), namely that a lobular tumour type is positively correlated to the number of children and age at first child $(\mathrm{p}=0.022)$. Lymphovascular invasion $(\mathrm{p}=0.041)$ and non-diploidy $(\mathrm{p}=0.038)$ (poor prognostic markers) are significantly correlated to low age at first child, $<23$ years. Similarly, estrogen negative tumors $(\mathrm{p}=0.011)$ are more common amongst women with a short time between last birth and time of diagnosis ( $\leq 26 \mathrm{yrs}$ ).

\section{Discussion}

We found a significant association between hormone use and lymphovascular invasion but no other associations between prognostic markers and hormone use. This is given support by recent research showing that the more hormones a woman consumed the more aggressive was the breast cancer developed, which may be explained by the fact that estradiol stimulate angiogenesis (Stoner et al., 2004; Seeger et al., 2006; Loof-Johanson et al., 2011; Caliceti et al., 2013).

Lymphovascular invasion that can be seen early in the periphery of a breast tumor can be viewed as a marker on angiogenesis / neovascularisation started up in the tumor considers Widodo et al., 2013. Endothelial growth factor (VEGF) can be a key mediator in tumour angiogenesis according Stoner et al., 2004; Garvin et al., 2005; Widodo et al., 2013; Huang et al., 2014. Tamoxifen, which is a selective estrogen receptor modulator, for the treatment of breast cancer has the opposite effect compared with estrogen. In other words - HRT and OC seem to provide an angiogenesis in the tumor while tamoxifen causes the 
opposite (Garvin et al., 2005; Huang et al., 2014). LVI is a predictor of recurrence and survival in the disease (Sanchez et al., 2010; Zhao et al., 2012; Scoppmann et al., 2013) and thus an important prognostic marker. Our study shows a significant link between the use of hormones and lymphovascular invasion of tumour cells.

Limitations of the study: Data on hormone use is based on interview responses and validation has not been made against patient records. Oral contraceptives were introduced in Sweden in 1964. Until 1985 the use of HRT was restricted due to side effects but later it became more common. The aim was to study breastfeeding combined with the consumption of oestrogen, gestagen, or a combination of these, to established prognostic markers but the concentrations of oestrogen has varied substantially over the study period. The patients in our material received diagnoses between 1988 and 1990.

The response rate of $72 \%$ is reasonable in a followup study over 20 years. Many responders were very old, affected by dementia or under terminal care. In some cases relatives have informed us of their inability to answer questions. Nevertheless, since childbirth and breastfeeding are important emotional experiences in a woman's life most patients thoroughly described breastfeeding times for their different children and this phenomenon has previously been observed (Loof-Johanson et al., 2010). Concerning hormone use, however, recollection is more uncertain.

In conclusions: $i$ ). Hormone use is significant linked to lymphovascular invasion, LVI, in breast cancer thereby affecting the prognosis of the disease, ii) Hormone use with breastfeeding does not interact with the prognostic variables presented, iii) Breastfeeding and the use of OC and HRT ought to be included in the preventive work against breast cancer

\section{Acknowledgements}

The authors declare that they have no competing interests. This work is supported by grants from the Research Council of Southern Sweden (FORSS).The funding sources had no role in study design, data collection and analysis, or interpretation and presentation of the results.

\section{References}

Anothaisintawee T, Wiratkapun C, Lerdsitthichai, P et al (2013). Risk factors of breast cancer: A systematic review and metaanalysis. Asia Pac J Public Health, 25, 368-87.

Antoine C, Ameye L, Paesmans M, et al (2014). Systematic review about breast cancer incidence in relation to hormone replacement therapy use. Climacteric, 17, 116-32.

Caliceti C, Aquila G, Pannella M, et al (2013). 17 beta-estradiol enhances signalling mediated by VEGF-A-delta-like ligand 4-notch1 axis in human endothelial cells. PloS One, 8, 1440.

Collaborative Group on Hormonal Factors in Breast Cancer: breast cancer and breastfeeding: collaborative reanalysis on individual data from 47 epidemiological studies in 30 countries, including 50302 women with breast cancer and 96973 Women Without the disease. (2002). The Lancet. 360, No 9328.
Dieterich B, Albe X, Vassilakos P, et al (1995). The prognostic value of DNA ploidy and S-phase estimate in primary breast cancer: a prospective study. Int J Cancer, 63, 49-54.

Ellis IO, Galea M, Broughton N, et al (1992). Pathological prognostic factors in breast cancer. II. Histological type. Relationship with survival in a large study with long-term follow-up. Histopathol, 20, 479-89.

Elston CW, Ellis IO, (1991). Pathological prognostic factors in breast cancer. I. The value of histological grade in breast cancer: experience from a large study with long-term followup. Histopathol, 19, 403-10.

Garvin S, Nilsson UW, Dabrosin C, (2005). Effects of oestradiol and tamoxifen on VEGF, soluble VEGFR-1 and VEGFR-2 in breast cancer and endothelial cells. Br J Cancer, 93, 1005-10.

Huang TS, Chen YJ, Chou Ty, et al (2014). Oestrogen-induced angiogenesis promotes adenomyosis by activating the SlugVEGF axis in endometrial epithelial cells. J Cell Mol Med, 18, 358-71.

Kallioniemi OP, Bianco G, Alavaikko M, et al (1987).Tumour DNA ploidy as an indenpendent prognostic factor in breast cancer. Br J Cancer, 56, 637-42.

Kwan ML, Bernard PS, Kroenke CH, et al (2015). Breastfeeding, PAM50 tumor subtype, and breast cancer prognosis and survival. J Natl Cancer Inst, 107.

Li CI, Beaber EF, Tang MT, et al (2013). Reproductive factors and risk of estrogen receptor positive, triple-negative, and HER2-neu overexpressing breast cancer among women 20-44 years of age. Breast Cancer Res Treat, 137, 579-87.

Loof-Johanson M, Brudin L, Sundquist M, et al (2011). Breastfeeding and prognostic markers in breast cancer. Breast, 20, 170-5.

Phipps AI, Chlebowski RT, Prentice R, et al (2011). Reproductive history and oral contraceptive use in relation to risk of triplenegative breast cancer. J Natl Cancer Inst, 103, 470-7.

Pinder SE, Ellis IO, Galea M, et al (1994). Pathological prognostic factors in breast cancer. III. Vascular invasion: relationship with recurrence and survival in a large study with long-term follow-up. Histopathol, 24, 41-7.

Sanchez BC,Sundqvist M, Fohlin H, et al (2010). Prolonged tamoxifen treatment increases relapse-free survival for patients with primary breast cancer expressing high levels of VEGF. Eur J Cancer, 46, 1580-7.

Schneider AP, Zainer ZM, Kubat CK, et al (2014). The breast cancer epidemic: 10 facts. Linacre $Q, 81,244-77$.

Schoppmann SF, Jesch B, Zacherl J, et al (2013). Lymphangiogenesis and lymphovascular invasion diminishes prognosis in esophageal cancer. Surgery, 153, 526-34.

Seeger H, Wallwiener D, Mueck AO, et al (2006). Different effects of estradiol and various antiestrogens on TNF-alphainduced changes of biochemical markers for growth and invasion of human breast cancer cells. Life Sci, 78, 1464-8.

Stoner M, Wormke M, Saville B, et al (2004). Estrogen regulation of vascular endothelial growth factor gene expression in ZR-75 breast cancer cells through interaction of estrogen receptor alpha and SP proteins. Oncogene, 23, 1052-63.

Stål O, Brisfors A, Carstensen J, et al (1992). Relationships of DNA ploidy, S-phase fraction and hormone receptor status to tumor stage in breast cancers detected by population screening. the south-east breast cancer group. Int J Cancer, 51, 28-33.

Sundquist M, (2000). Prognostic factors in breast carcinoma: linkopings university, medical dissertations no 632.

Widodo I, Ferronika P, Harijadi A, et al (2013). Clinicopathological significance of lymphangiogenesis and tumor lymphovascular invasion in Indonesian breast cancers. Asian Pac J Cancer Prev, 14, 997-1001.

Work ME, John EM, Andrulis IL, et al (2014). Reproductive 
Margaretha Loof-Johanson et al

risk factors and oestrogen/progesterone receptor-negative breast cancer in the Breast Cancer Family Registry. $\mathrm{Br} J$ Cancer, 110, 1367-77.

Zhao YC,Ni XJ,LiY, et al (2012). Peritumoral lymphangiogenesis induced by vascular endothelial growth factor $\mathrm{C}$ and $\mathrm{D}$ promotes lymph node metastasis in breast cancer patients. World J Surg Oncol, 10, 165. 